\title{
A Full Snow Season in Yellowstone: A Database of Restored Aqua Band 6
}

\author{
Irina Gladkova, Michael Grossberg, George Bonev, Peter Romanov, George Riggs, and Dorothy Hall
}

\begin{abstract}
The algorithms for estimating snow extent for the Moderate Resolution Imaging Spectroradiometer (MODIS) optimally use the 1.6- $\mu \mathrm{m}$ channel which is unavailable for MODIS on Aqua due to detector damage. As a test bed to demonstrate that Aqua band 6 can be restored, we chose the area surrounding Yellowstone and Grand Teton national parks. In such rugged and difficult-to-access terrain, satellite images are particularly important for providing an estimation of snow-cover extent. For the full 2010-2011 snow season covering the Yellowstone region, we have used quantitative image restoration to create a database of restored Aqua band 6 . The database includes restored radiances, normalized vegetation index, normalized snow index, thermal data, and band-6-based snow-map products. The restored Aqua-band-6 data have also been regridded and combined with Terra data to produce a snow-cover map that utilizes both Terra and Aqua snow maps. Using this database, we show that the restored Aqua-band-6-based snow-cover extent has a comparable performance with respect to ground stations to the one based on Terra. The result of a restored band 6 from Aqua is that we have an additional band-6 image of the Yellowstone region each day. This image can be used to mitigate cloud occlusion, using the same algorithms used for band 6 on Terra. We show an application of this database of restored band-6 images to illustrate the value of creating a cloud gap filling using the National Aeronautics and Space Administration's operational cloud masks and data from both Aqua and Terra.
\end{abstract}

Index Terms-Aqua band 6, Moderate Resolution Imaging Spectroradiometer (MODIS), normalized difference snow index, restoration.

\section{INTRODUCTION}

$\mathbf{T}$ HE area surrounding Yellowstone and Grand Teton national parks has a diversity of flora, fauna, local ecosystems, and land types such as mountains and subalpine forests.

Manuscript received April 25, 2012; revised July 2, 2012; accepted July 26, 2012. This work was supported in part by Center for Satellite Applications and Research, National Oceanic and Atmospheric Administration/ National Environmental Satellite, Data, and Information Service, under Grant DG133E07CQ0077. The works of D. Hall and G. Riggs were supported by the National Aeronautics and Space Administration's Earth Observing System Project. The views, opinions, and findings in this paper are those of the authors and should not be construed as an official NOAA and/or U.S. Government position, policy, or decision.

I. Gladkova, M. Grossberg, and P. Romanov are with Cooperative Remote Sensing Science and Technology Center, NOAA, New York, NY 10031 USA (e-mail: gladkova@cs.ccny.cuny.edu; grossberg@cs.ccny.cuny.edu; Peter. Romanov@noaa.gov).

G. Bonev is with The Graduate Center, City University of New York, New York, NY 10016 USA (e-mail: gbonev@ gc.cuny.edu).

G. Riggs and D. Hall are with the Cryospheric Sciences Laboratory, NASA Goddard Space Flight Center, Greenbelt, MD 20771 USA (e-mail: Dorothy.K.Hall@nasa.gov; george.a.riggs@nasa.gov).

Color versions of one or more of the figures in this paper are available online at http://ieeexplore.ieee.org.

Digital Object Identifier 10.1109/LGRS.2012.2213063
During fall and winter months, the area can receive a considerable amount of snow. While there are a number of snow stations in and around the parks, during winter, much of the area is difficult to access. Remote sensing data, such as the standard snow maps from the National Aeronautics and Space Administration (NASA) Moderate Resolution Imaging Spectroradiometer (MODIS) instruments [1], provide a way to study the buildup and depletion of the snow cover. The NASA standard MODIS snow algorithms use information from multiple bands of MODIS to map fractional snow cover and snow albedo [2], [3]. The algorithm was designed for use with both Terra and Aqua, but unfortunately, it cannot be applied directly to Aqua since it relies on band 6 , on which three-fourths of the detectors are dead or extremely noisy. As a result, the standard snow-cover algorithm for Aqua has been modified to use the 2.1- $\mu \mathrm{m}$ channel, band 7 [2], [4]. Although this works quite well, it produces different results which are thought to be inferior to results produced using the fully functioning band 6 [5].

Recently, we have developed a quantitative image restoration (QIR) technique [6]-[8] and applied it to MODIS/Aqua band 6 to produce an improved Aqua snow map [9]. The restoration of Aqua band 6 makes it possible to create two snow masks per day using the same algorithm used with Terra images. This provides more consistent results when comparing pixels from the same day. The one advantage is that we can use the second image to mitigate obscuration by clouds. In addition, it can also be used to account for differences in lighting due to shadows from the mountains. We created a database of restored Aqua band 6 over the Yellowstone region for the 2010-2011 snow season [10] to evaluate the benefit of band-6 restoration for snow products. In this letter, we describe this database, which fills in some of that record which had been previously assumed lost.

Previously, much work has been conducted to combine the Terra and Aqua standard snow maps to produce clearer views of snow cover (see, for example, [11]-[13] for improved daily snow-cover mapping). In addition, there is a cloud-gap-filled (CGF) product developed to provide clear daily views of snow cover [14]. Rather than use a band-7-based snow product for Aqua, we propose utilizing the QIR technique, so that the same snow algorithm can be used for both Terra and Aqua to map snow cover. We note that we make no modifications to NASA's operational cloud mask and the snow extent algorithm is only applied to the clear-sky pixels. We validated the computed snow product against measurements from 76 ground stations (available from [15]).

Using the same algorithm on both the MODIS Terra and Aqua to monitor snow-cover change will enable the analysis of snow-cover variability over seasons and years. It will also 


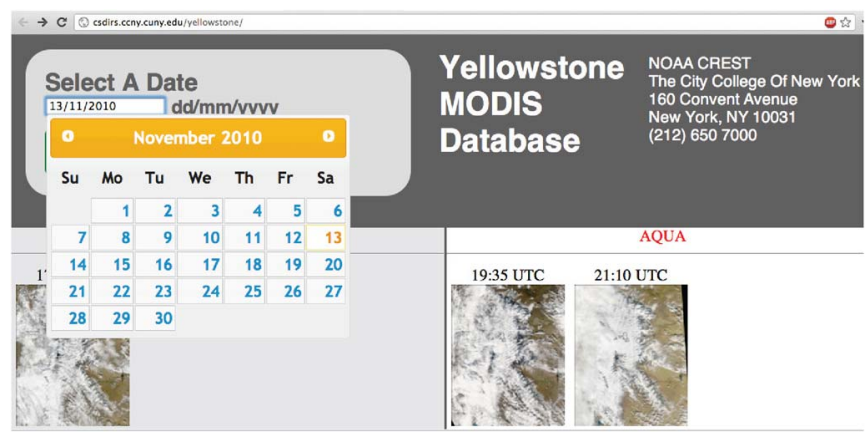

Fig. 1. Screen shot of the Yellowstone MODIS database. Once a date is selected, the search will show all the granules which intersect the region of interest on the given day. Clicking on a granule leads to a page where all the products can be downloaded.

contribute to the understanding of the complex relationship between snow cover and stream flow in the context of climate change through the study of changes in stream flow related to snow cover. Such information may be used to develop snowcover depletion curves to study changing water resources in the context of climate change [16] in the Yellowstone region.

While the main focus of this letter is to report on our database of the Aqua-based snow mask, the restored Aqua-band-6 data have other potential applications as well. MODIS Terra band 6 is used in the algorithm for determining cloud pixels, and it is also used in products that measure aerosol. By making the restored Aqua band 6 available in our database, researchers can consider the addition of a second band- 6 image for other applications. A web-based interface to our database is available at [10].

\section{Description OF THE DATABASE}

The products in our database (available at http://csdirs.ccny. cuny.edu/yellowstone/) can be selected by time. In constructing our database, we focused on the 2010-2011 snow season. The snow season was assumed to start on August 15, 2010, and end on May 22, 2011, spanning 281 days. During this time interval, we have restored band 6 for all Aqua granules for which the field of view of MODIS intersects a box between $42.0^{\circ} \mathrm{N}$ and $45.8^{\circ} \mathrm{N}$ latitude and $-112.2^{\circ} \mathrm{E}$ and $-108.0^{\circ} \mathrm{E}$ longitude. This area includes the Greater Yellowstone region. Along with the restored radiances, the database provides the normalized difference vegetation index, normalized difference snow index, thermal image, and band-6-based snow-map product. In addition, we have regridded the restored Aqua based on restored band 6 and combined Aqua and Terra data to produce a Terra-Aqua combined snow-cover map product for the snow season.

The restoration algorithm is based on a local multilinear regression over spatial/spectral windows using bands 1 through 7 as well as the good detectors of band 6 as training data. This algorithm was previously described in detail in [8] and implemented in Python using the NumPy, SciPy, and Matplotlib libraries. The metadata for the database are maintained in a relational PostgreSQL database, and the web interface was developed using Python/Django and Javascript/JQuery. The screen capture of the database is shown in Fig. 1.

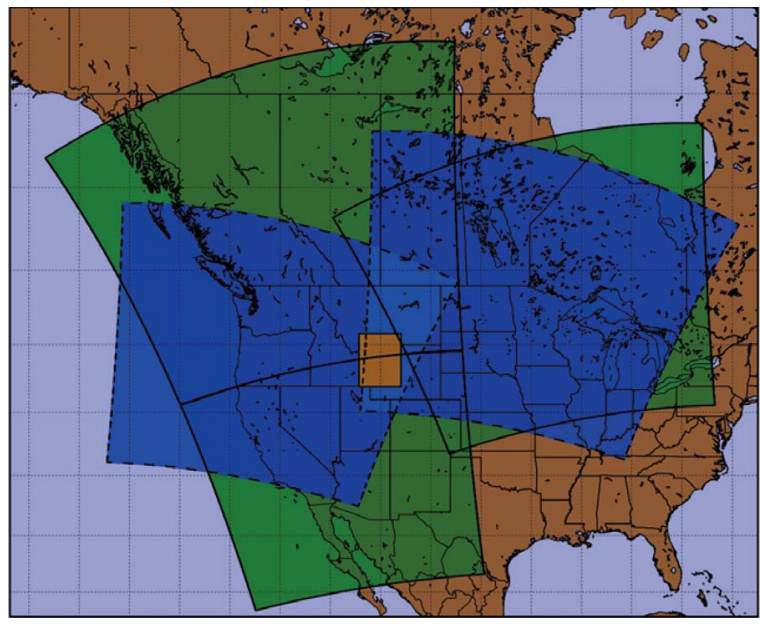

Fig. 2. Geolocation of all MODIS granules for one particular day (October 16, 2010) with respect to the Yellowstone area. (Blue) Terra had two granules and (green) Aqua had three granules that intersected the (orange) region of interest.

The database contains 474 Terra granules and the same number of Aqua granules. The number of granules per satellite in the database on a given day depends on the overpass and varies from one to three. The variation is shown in Fig. 2 which shows the fields of view of granules from Terra (blue) and Aqua (green) on October 16, 2010, and the region of interest (orange). The region of interest intersects two Terra and three Aqua granules on that day. The mean time of the Terra overpass is 20:17 Coordinated Universal Time (UTC) (1:17 P.M. local time), and the mean time of the Aqua overpass is 22:22 UTC (3:22 P.M. local time).

\section{Database Highlights}

Even though the database contains a limited region and spans a single season, it is already possible to apply it to a number of evaluation problems. To evaluate the use of the QIR Aqua band 6 in the snow product, we computed a confusion matrix using ground stations for both Terra and the QIR Aqua. The snowdepth measurements used as ground truth were obtained from the National Water and Climate Center [15]. We have not used the snow-depth values since the ground-station measurements were only used to identify the presence of snow. The overall accuracy for Terra is 0.96 , and the overall accuracy for Aqua (using our restored band 6) is 0.95 . The accuracy was calculated for clear-sky pixels only. Fig. 3 shows the season averaged accuracy for the Terra (shown in brown bars) and the QIRbased Aqua (shown in blue bars) snow mask at each of the 76 stations in the region. The confusion matrix for Terra and the QIR-restored Aqua is shown in Table I. As can be seen in Table I, the total number of measurements for Aqua and Terra are quite different. It is important to emphasize that the standard NASA cloud product uses band 6 in Terra but not in Aqua due to the damage. This may contribute to the increased number of cloud pixels in Aqua product. Nevertheless, it is clear from both the graph and the confusion matrix that the performance of the Terra and the QIR Aqua based snow masks are comparable. We have also compared a QIR band-6-based snow product to 


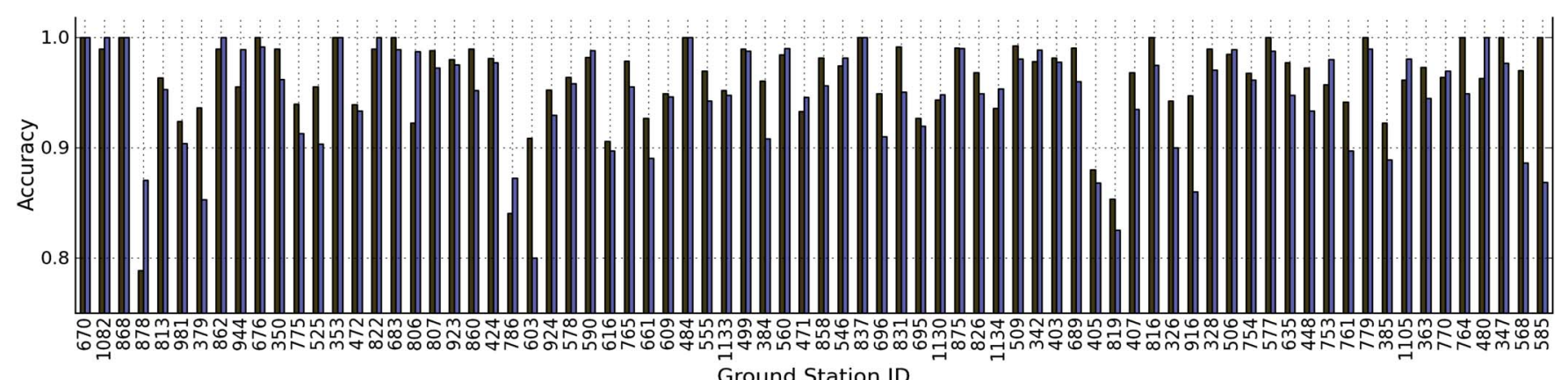

Fig. 3. Accuracy values for each of the 76 ground stations are depicted in the bar plot above. The brown bars correspond to Terra and the blue bars correspond to restored Aqua.

TABLE I

CONFUSION MATRIX

\begin{tabular}{|l|l||l|l|}
\hline \multicolumn{2}{|c||}{ Terra } & \multicolumn{2}{c|}{ Aqua } \\
\hline \hline TP: 3733 & FN: 297 & TP: 2347 & FN: 325 \\
$44.4 \%$ & $3.5 \%$ & $34.37 \%$ & $4.76 \%$ \\
\hline FP: 31 & TN: 4346 & FP: 46 & TN: 4111 \\
$0.38 \%$ & $51.7 \%$ & $0.67 \%$ & $60.2 \%$ \\
\hline \multicolumn{2}{|l|}{ Total: 8408} & Total: 6829 \\
\hline
\end{tabular}

Accuracy in terms of True Positives (TP), False

Positives (FP), True Negatives (TN), False Negatives (FN)

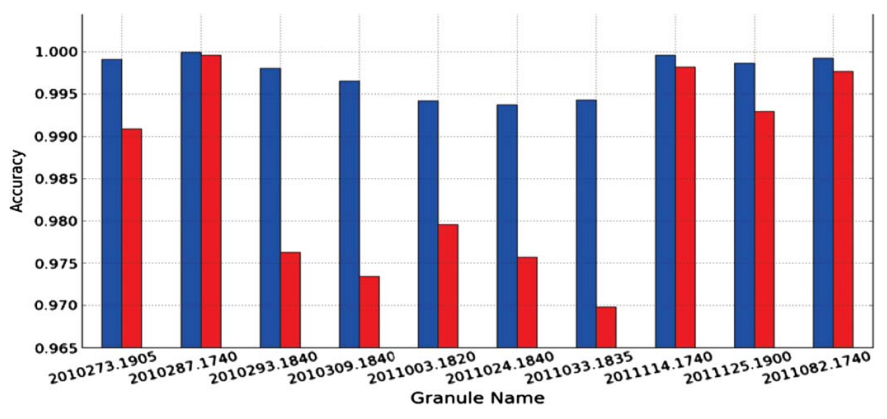

Fig. 4. Accuracy values computed for ten representative granules restricted to the Yellowstone region. The (blue) restored band 6 has improved accuracy (particularly in winter months) over the (red) band 7.

the Terra band-7-based snow product, where we simulated the Aqua-like damage to band 6. Evaluation was performed on a representative set of granules from one snow season. Using the QIR-restored band 6 compared with band 7 greatly improved accuracy referenced to an original band-6 product (cf. Fig. 4). This is particularly true for the winter months, as seen in the middle five bars of Fig. 4.

In addition to evaluation of the restored band 6, the database we have created is also useful in developing a combined CGF product that uses two snow masks per day to help mitigate cloud cover. To assign a meaningful snow flag when the surface is occluded by clouds, the CGF snow product uses the last available flag value [14]. Using Terra and Aqua independently reduces the number of cloud-free measurements available as shown in Fig. 5. The figure shows snow-depth measurements from ground station identifier (ID) 924, West Yellowstone (curve at the bottom), and the georegistered snow masks from Aqua and Terra (bars at the top). According to ground-station measurements, November had snowfall, but using Terra, there was continuous occlusion by clouds, as indicated by the pink shading for the time interval. As a result, the CGF mask, which is based only on Terra, records no snow through late November, indicated by a brown colored interval. By using the QIR-restored Aqua for cloud gap filling, we are able to detect snow, represented by cyan, nearly three weeks earlier. While snow depth at a ground station may not always indicate snow throughout a pixel, by using the restored band 6 , the CGF values are in better agreement with the ground measurements. Such a potential improvement to CGF snow mask made possible by the addition of Aqua-based cloud mask using QIR is shown in the fourth bar from the top.

We have also computed the mean cloud coverage for the entire snow season based on the cloud mask from the level-2 version-5 snow-cover product MOD10_L2 [2]. The percentage of cloud flags over the span of 281 consecutive days for both Aqua and Terra is shown in Fig. 6. Pixels that had more than one observation on a given day were included in the cloud coverage count only if all of the observations for that day had a cloud flag. As can be seen in Fig. 6, the cloud coverage over the mountains is much higher than for other regions.

There is also a significantly higher percentage of cloud pixels for Aqua than for Terra, even though the passes are roughly only $2 \mathrm{~h}$ apart. One possible explanation is that snow is sometimes misclassified as cloud, particularly for Aqua, because band 6 is a particularly valuable asset in distinguishing cloud from snow due to the fact that snow is darker than clouds in this band. The left panel in Fig. 6 shows the percentage of cloud cover (calculated using band 6) over the snow season of 281 consecutive days using MODIS Terra. When that band is not available, band 7-from which it is more difficult to distinguish clouds from snow-is relied upon. It is interesting to consider that, for ground-station locations which report snow, red dots shown on the color image in the middle of Fig. 6, there is a dramatic increase in the corresponding pixels indicating cloud in the cloud mask, when comparing Terra and Aqua on the far left and on the far right of Fig. 6, respectively. To further clarify this, an apparently clear-sky day, March 23, 2011, is shown in Fig. 7. On the far left, the Terra-based snow mask shows snow on the mountains with clouds along the boundary between snow and snow-free land. Despite apparently nearly identical and largely cloud-free RGB images for Terra (17:40 UTC) and Aqua (21:00 UTC), the number of pixels identified as cloud in the Aqua cloud mask has dramatically increased. It is possible that using a QIR-based cloud mask for Aqua may give better agreement with the Terra standard cloud mask. 

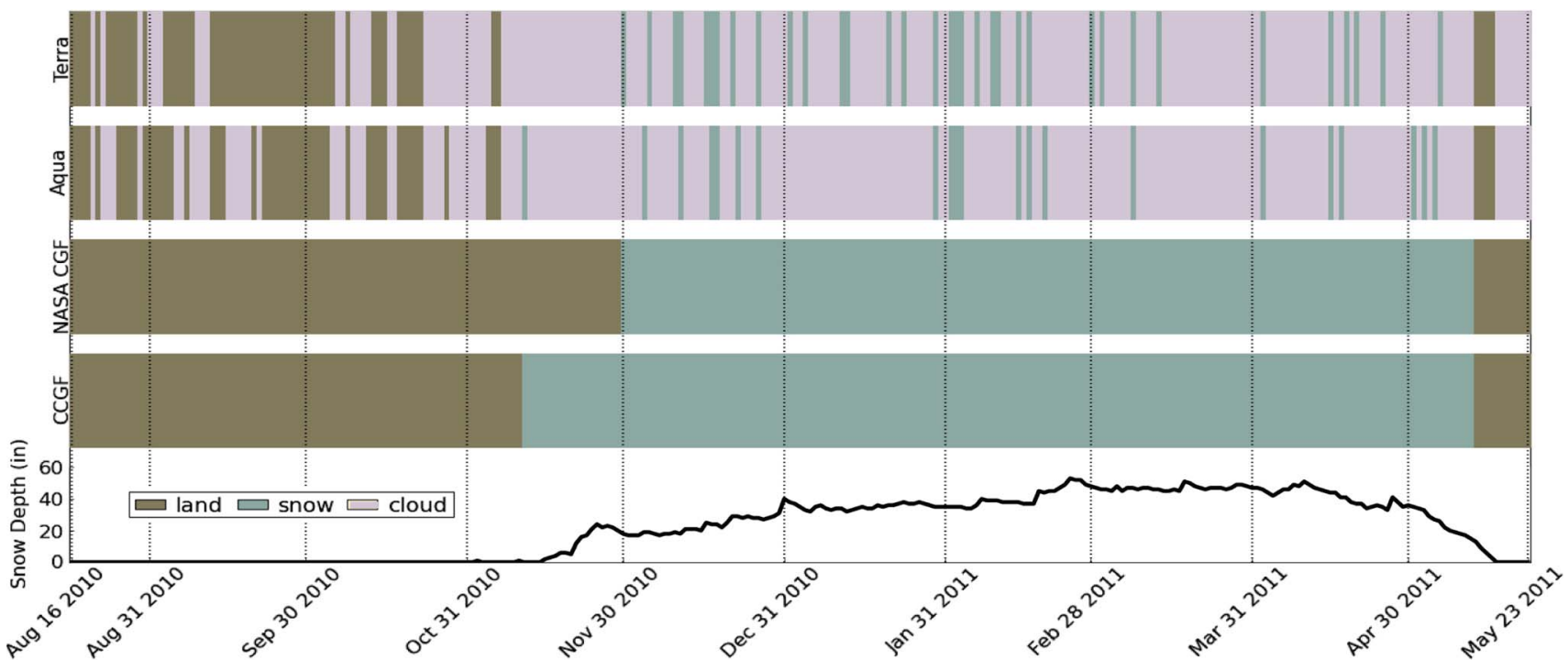

Fig. 5. Illustration of the potential improvement to CGF snow mask made possible by the addition of the Aqua-based cloud mask using QIR. From top to bottom, the bars display the Terra snow mask, QIR-based Aqua snow mask, current CGF Terra, and Terra-and-QIR/Aqua combined snow mask. In the bars, brown is snow-free land, pink is cloud covered, and cyan is snow, which is detected nearly three weeks earlier using combined-Terra-and-QIR/Aqua-based cloud gap filling. The graph corresponds to measurements recorded at West Yellowstone ground station (ID 924).
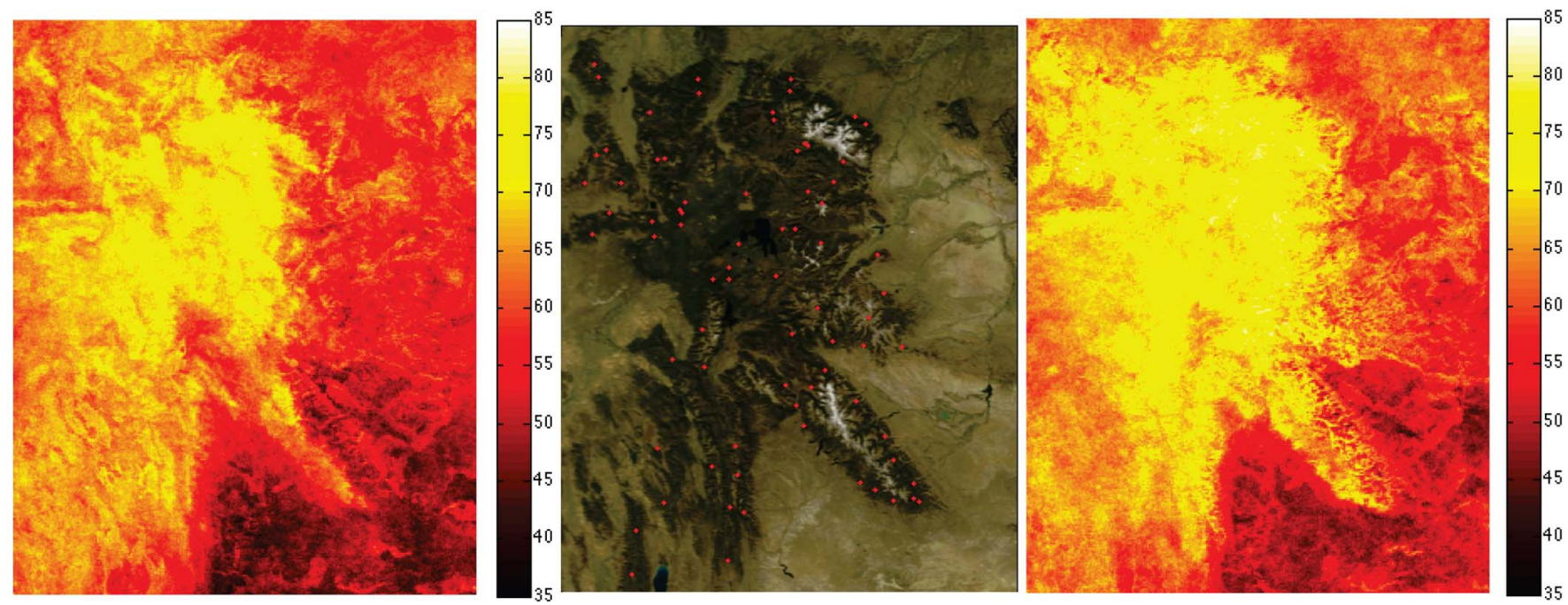

Fig. 6. Percent of cloud coverage over 281 consecutive days for (left) Terra and (right) Aqua. The RGB image of the (middle) Yellowstone region with red dots corresponding to the locations of ground stations used in this letter as georeferences. The great increase in locations that ground stations report as having snow and that are identified in the Aqua mask as being cloud covered may partially be due to the loss of band 6, which is important for distinguishing cloud from snow.
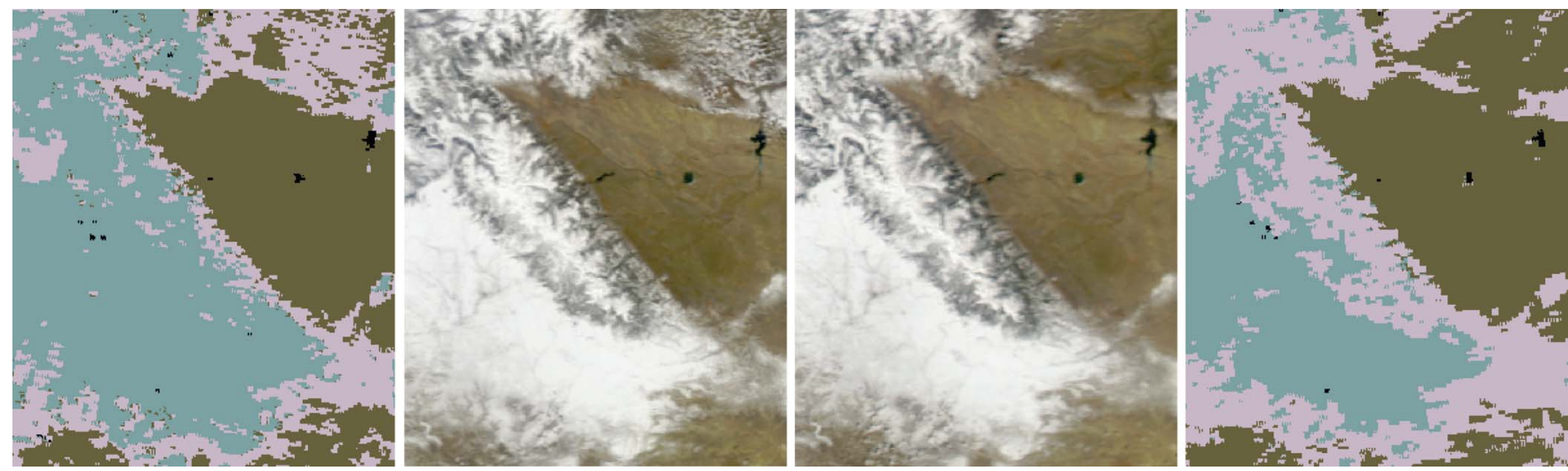

Fig. 7. Example of misclassifying snow as cloud. All images are for March 23, 2011, which appears to have a nearly clear sky when looking at the RGB images for Aqua and Terra. (From left to right) Terra snow mask, Terra RGB, Aqua RGB, and Aqua snow mask. Cyan is used for snow, brown represents snow-free land, pink represents clouds, and black indicates other flags corresponding to missing pixels, saturated, no decision, and inland water (including lake ice). 


\section{CONClusions And Future Work}

We have created a database of restored MODIS/Aqua band 6 using QIR for one snow season over the Yellowstone region. Using a random subsample, we have used Terra with simulated damage to show that using restored band 6 is more accurate than using band 7. Using ground stations over the same region, we show that we are able to achieve comparable accuracy for Terra and restored Aqua. We have shown that, by having both Terra and Aqua measurements available, cloud gap filling can be improved, in some cases, showing the presence of snow weeks earlier. Finally, there are indications that the lack of band 6 in Aqua degrades the ability to distinguish snow from clouds, which may indicate an opportunity for a restored band 6 to be used in the cloud masks.

\section{ACKNOWLEDGMENT}

The authors would like to thank P. Alabi for helping them retrieve data and $\mathrm{H}$. Aizenman for editing and help with figures.

\section{REFERENCES}

[1] [Online]. Available: http://modis-snow-ice.gsfc.nasa.gov/

[2] D. K. Hall, G. A. Riggs, V. V. Salomonson, N. E. DiGirolamo, and K. A. Bayr, "MODIS snow-cover products," Remote Sens. Environ., vol. 83, no. 1/2, pp. 181-194, Nov. 2002.

[3] G. A. Riggs, D. K. Hall, and V. V. Salomonson, MODIS Snow Products User Guide, MODIS NASA Gov., Washington, DC. [Online]. Available: http://modis-snow-ice.gsfc.nasa.gov/uploads/sug_c5.pdf
[4] G. A. Riggs and D. K. Hall, "Snow mapping with the MODIS Aqua instrument," in Proc. 61st Eastern Snow Conf., 2004, pp. 81-84.

[5] D. Hall and G. Riggs, "Accuracy assessment of the MODIS snow products," Hydrol. Process., vol. 21, no. 12, pp. 1534-1547, Jun. 2007.

[6] I. Gladkova, M. Grossberg, and F. Shahriar, "Quantitative image restoration," in Proc. SPIE, Algorithms Technol. Multispectral, Hyperspectral, Ultraspectral Imagery, 2010, vol. 7695, pp. 769519-1-769519-12.

[7] I. Gladkova, M. Grossberg, G. Bonev, and F. Shahriar, "A multiband statistical restoration of the Aqua MODIS 1.6 micrometer band," in Proc. SPIE, 2011, vol. 8048, pp. 1-12.

[8] I. Gladkova, M. Grossberg, F. Shahriar, G. Bonev, and P. Romanov, "Quantitative restoration for MODIS band 6 on Aqua," IEEE Trans. Geosci. Remote Sens., vol. 50, no. 6, pp. 2409-2416, Jun. 2012.

[9] I. Gladkova, M. Grossberg, G. Bonev, P. Romanov, and F. Shahriar, "Increasing the accuracy of MODIS/Aqua snow product using quantitative image restoration technique," IEEE Geosci. Remote Sens. Lett., vol. 9, no. 4, pp. 740-743, Jul. 2012.

[10] [Online]. Available: http://csdirs.ccny.cuny.edu/csdirs/yellowstone

[11] J. Parajka and G. Blöschl, "Spatio-temporal combination of MODIS images potential for snow cover mapping," Water Resources Res., vol. 44, no. 3, pp. W03406-1-W03406-13, Mar. 2008.

[12] X. Wang and H. Xie, "New snow cover products from combining MODIS Terra and Aqua observations," Comput. Geosci., to be published.

[13] X. Wang, H. Xie, T. Liang, and X. Huang, "Comparison and validation of MODIS standard and new combination of Terra and Aqua snow cover products in northern Xinjiang," China: Hydrol. Process., vol. 23, no. 3, pp. 419-429, Jan. 2009.

[14] D. K. Hall, G. A. Riggs, J. L. Foster, and S. Kumar, "Development and validation of a cloud-gap filled MODIS daily snowcover product," Remote Sens. Environ., vol. 114, no. 3, pp. 496-503, Mar. 2010.

[15] [Online]. Available: http://www.wcc.nrcs.usda.gov/

[16] D. K. Hall, J. L. Foster, N. E. DiGirolamo, and G. A. Riggs, "Snow cover, snowmelt timing and stream power in the Wind River Range," Geomorphology, vol. 137, no. 1, pp. 87-93, Jan. 2012. 